\title{
A critical role of AREG for bleomycin-induced skin fibrosis
}

\author{
Mary Yinghua Zhang' ${ }^{1}$, Shuyi Fang ${ }^{2}$, Hongyu Gao ${ }^{3}$, Xiaoli Zhang ${ }^{1}$, Dongsheng Gu' , Yunlong Liu 2,3,4,5, \\ Jun Wan $2,3,4,5$ and Jingwu Xie ${ }^{1,3^{*}}$ (1)
}

\begin{abstract}
We report our discovery of an important player in the development of skin fibrosis, a hallmark of scleroderma. Scleroderma is a fibrotic disease, affecting 70,000 to 150,000 Americans. Fibrosis is a pathological wound healing process that produces an excessive extracellular matrix to interfere with normal organ function. Fibrosis contributes to nearly half of human mortality. Scleroderma has heterogeneous phenotypes, unpredictable outcomes, no validated biomarkers, and no effective treatment. Thus, strategies to slow down scleroderma progression represent an urgent medical need. While a pathological wound healing process like fibrosis leaves scars and weakens organ function, oral mucosa wound healing is a scarless process. After re-analyses of gene expression datasets from oral mucosa wound healing and skin fibrosis, we discovered that several pathways constitutively activated in skin fibrosis are transiently induced during oral mucosa wound healing process, particularly the amphiregulin (Areg) gene. Areg expression is upregulated $\sim 10$ folds $24 \mathrm{hrs}$ after oral mucosa wound but reduced to the basal level 3 days later. During bleomycininduced skin fibrosis, a commonly used mouse model for skin fibrosis, Areg is up-regulated throughout the fibrogenesis and is associated with elevated cell proliferation in the dermis. To demonstrate the role of Areg for skin fibrosis, we used mice with Areg knockout, and found that Areg deficiency essentially prevents bleomycin-induced skin fibrosis. We further determined that bleomycin-induced cell proliferation in the dermis was not observed in the Areg null mice. Furthermore, we found that inhibiting MEK, a downstream signaling effector of Areg, by selumetinib also effectively blocked bleomycin-based skin fibrosis model. Based on these results, we concluded that the Areg-EGFRMEK signaling axis is critical for skin fibrosis development. Blocking this signaling axis may be effective in treating scleroderma.
\end{abstract}

Keywords: Scleroderma, Skin fibrosis, Bleomycin, Areg, MEK

\section{Background}

Scleroderma affects 70,000 to 150,000 Americans [1, 2]. Due to the heterogeneous phenotypes, unpredictable outcomes, no validated biomarkers, and no effective treatment, scleroderma is a very hard disease to manage in the clinic. Similar to other fibrotic diseases, scleroderma is a pathological wound healing process that

\footnotetext{
*Correspondence: jinxie@iu.edu

1 Department of Pediatrics, The Wells Center for Pediatric Research, Indiana University School of Medicine, Indianapolis, IN, USA

Full list of author information is available at the end of the article
}

produces an excessive extracellular matrix to interfere with normal organ function [3-6]. Fibrosis is known to contribute to nearly half of human mortality [7]. While molecular mechanisms underlying fibrosis remain elusive, fibrosis shares several commons processes. Chronic injury in tissues is the initial trigger for fibrosis, which draws inflammatory responses [3]. Effector cells, such as fibroblasts, become activated in order to repair tissue damages. During the repair process, extracellular matrix (ECM) is produced. Unlike scarless wound repair in oral mucosa [8], fibrosis is caused by excessive ECM production from over-activated effector cells. As a result, fibrotic 
tissues are formed, leading to abnormal tissue function or even organ failure.

One critical step in fibrosis is production of excessive ECM from over activated effector cells. In contrast to fibrosis, oral mucosa wound healing has less ECM and controlled number of effector cells [9]. We hypothesize that by comparing scarless oral mucosa wound healing with skin fibrosis on gene expression, we may find molecules that are critical for scleroderma development [8]. Since there are many studies on the time course of gene expression during oral mucosa wound healing and skin fibrosis, we took the advantage of public datasets to reanalyze gene expression during oral mucosa wound healing. Specifically, we are interested in those genes with transient induction after injury in oral mucosa [9]. These genes will be further analyzed for their expression at different time points during skin fibrosis.

We found that Areg and the ErbB signaling pathway is transiently induced during oral mucosa wound healing. In contrast, Areg is highly expressed throughout the process of skin fibrosis. Using Areg knockout mice and a specific inhibitor of downstream effector MEK, selumetinib, we demonstrated that the Areg-EGFR-MEK signaling axis is critical for bleomycin-induced skin fibrosis.

\section{Results}

Gene expression comparison between oral wound and skin fibrosis

We hypothesized that the genes, that are transiently induced during oral mucosa wound healing but upregulated during skin fibrosis, may be critical for driving skin fibrosis. Several public datasets are available on gene expression during oral cavity wound healing, skin wound and skin fibrosis. We chose to use GSE23006 in which gene expression data from multiple time points during oral wound healing are available $[9,10]$. GSE132869 (from Geo datasets) was used for re-analyses of gene expression during bleomycin-induced skin fibrosis.

In analyses of GSE23006, we focused on the genes induced $12 \mathrm{hr}$ and $24 \mathrm{hrs}$ after wound but returned to the basal level $( \pm 20 \%) 3$ days after injury. Only genes induced over 2 folds after injury were further analyzed for their expression in bleomycin-mediated skin fibrosis. As shown in Additional file: 1, 91 genes were induced shortly after injury but returned to the basal level 3 days later. Gene ontology analysis indicates that two of these genes are ErbB-related molecules AREG and HBEGF (Additional file: 1, Fig. 1a). Others include molecules related to immunity, keratinization, antivirus defense and chemotaxis (Fig. 1a).

We further analyzed expression of these genes in GSE132869, and 9 genes were up-regulated in bleomycininduced skin fibrosis (Additional file: 2). These molecules include molecules known to be involved in fibrosis, such as Mmp9, Cxcl1, Ccl5, Fosl1 and Stat1. For example, Mmp9 is known to be important for skin fibrosis [11-13]. The function of other 3 genes, Klra2, Slfn4 and $H d c$, during fibrosis is not known. In contrast, the pattern of Areg expression is very intriguing. Areg was induced to $\sim 10$ folds shortly after oral cavity wound, returned to the basal level 3 days later (Fig. 1b), but was up-regulated throughout bleomycin-induced skin fibrosis (Additional file: 2, Fig. 1c). We have confirmed elevated Areg expression during skin fibrosis using skin specimens at different time points (Fig. 1d). Since Areg is a known growth factor with well-characterized signaling downstream effectors (Areg-EGFR-RAS-RAF-MEK), we focused our efforts to determine the role of Areg signaling for bleomycin-mediated skin fibrosis.

\section{Skin fibrosis in Areg null mice}

Because Areg is one of the few genes with transient induction during oral wound healing but maintains high expression throughout skin fibrosis, we used Areg knockout mice to determine the significance of Areg for bleomycin-induced fibrosis.

We used skin thickness as a way to measure skin fibrosis. Skin thickening is one of the earliest manifestations of scleroderma; and is one of the most widely used measures in the clinical trials [14-17]. Several studies have demonstrated that the extent of skin involvement directly correlates with internal organ involvement and prognosis in scleroderma patients.

We obtained Areg knockout mice and wild type mice to perform bleomycin-induced skin fibrosis through daily intradermal injection of bleomycin $(50 \mu \mathrm{l}$ of $1 \mathrm{mg} / \mathrm{ml}$ bleomycin in PBS) for 10 days. At the end of the study, skin biopsies at the injected site or at a site far away the injection site were harvested to perform H\&E staining, and the skin thickness, which is the combination of epidermis and dermis, was measured by Image J. In wild type mice, bleomycin injection increased the skin thickness $\sim 80-150 \%$ (Fig. 2, P < 0.001). In Areg null mice, however, we only observed $<20 \%$ increase of skin thickness after intradermal injection of bleomycin. The data from gene knockout mice demonstrates that Areg is critical for skin fibrosis.

We used Fast green/ Sirius red staining to confirm fibrosis in the skin, with Sirius red stains collagen proteins whereas Fast green stains non-collagen proteins (Additional file: 3). We found that bleomycin treatment increased Sirius red staining, less red staining was observed in Areg knockout mice.

Increased proliferation of fibroblasts and other cell types is accompanied with fibrosis, and the level of cell proliferation has been used to monitor disease 


$$
\begin{array}{|l|}
\hline \text { Immunity } \\
\text { Innate immunity } \\
\text { Keratinization } \\
\text { Antiviral defense } \\
\text { EGF-like domain } \\
\text { Chemotaxis } \\
\text { Glycoprotein } \\
\text { Growth factor }
\end{array}
$$

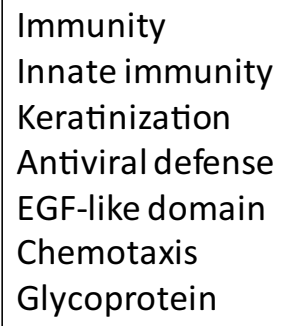$$
\text { a - Summary of gene ontology analysis of up-regulated }
$$$$
\text { genes in oral mucosa wound healing }
$$

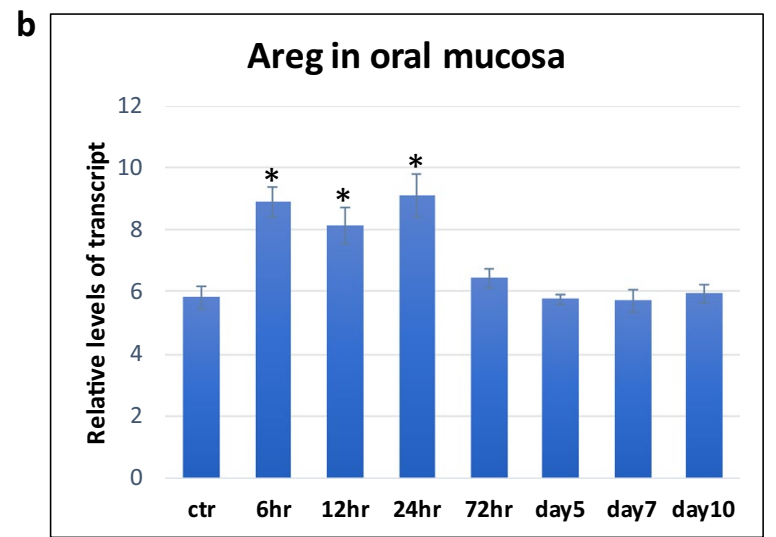

c



d

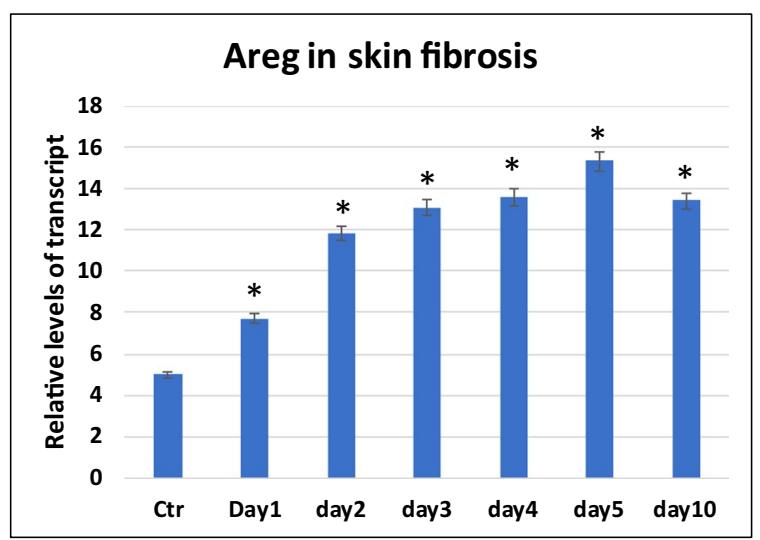

Fig. 1 Areg expression in oral wound healing and skin fibrosis. a shows a summary of ontology analysis of transient up-regulated genes during oral mucosa wound healing. The list of genes with transient induction is shown in Additional file 1: Table S1. b shows a re-analysis of Areg expression during oral mucosa wound healing 8 , and * indicates a significant change $(p<0.01)$. c shows re-analysis of Areg gene expression from GSE132869 (from Geo datasets), confirming the significant expression in bleomycin-induced skin fibrosis. Each dot represents one sample. PBS was used as a control, and BLM indicates bleomycin treatment. $\mathbf{d}$ shows time-dependent expression of Areg during bleomycin-induced skin fibrosis. Please note that Areg was consistently highly expressed in the skin biopsies after bleomycin treatment at different time points. ${ }^{*}$ indicates a significant change $(p<0.01)$

progression or treatment outcomes in the mouse models [18-20]. We detected expression of Ki-67 in skin specimens following bleomycin injection to determine the specific effects on cell proliferation and fibrosis progression in mice. We detected an increase in Ki-67 positivity in dermis of bleomycin-injected mice (Fig. 3), indicating an elevated cell proliferation during skin fibrosis. Our data are consistent with previous studies to show elevated cell proliferation during fibrosis [21]. In Areg null mice, however, bleomycin did not increase the level of Ki-67 positivity in skin dermis, suggesting a role of Areg for cell proliferation in skin dermis during fibrosis (Fig. 3).

\section{Blocking MEK signaling prevents bleomycin-induced skin fibrosis}

There are several downstream effectors of Areg, including the receptor EGFR, MEK and PI3K. Selumetinib (also AZD6244) is a specific MEK inhibitor, now approved by FDA to treat children 2 years and older with neurofibromatosis type I [22-24]. We hypothesized that if MEK mediates Areg effects in skin fibrosis, treatment of selumetinib should reduce bleomycin-mediated skin fibrosis.

First, we determined that MEK is activated in skin fibrosis. Using specific antibodies to phospho-ERK, we found that a high percentage of phospho-ERK positive cells in the skin from mice treated with bleomycin (Fig. 4). Next, we treated mice with selumetinib (oral gavage, $15 \mathrm{mg} / \mathrm{kg}$ / every other day), together with intradermal injection of bleomycin ( 50 microL of $1 \mathrm{mg} / \mathrm{ml}$ bleomycin in PBS) for 2 weeks and determined skin thickness after H\&E staining of the skin biopsies. As shown in Fig. 5, we found that while wild type control skin increased its thickness over $80 \%$ after bleomycin treatment, selumetinib treated skin tissues had $<10 \%$ of increase in skin thickness. We also confirmed skin fibrosis by Fast green/ Sirius red staining by showing that treatment with selumetnib (AZD6244) led to less Sirius red staining (Additional file: 3). This 

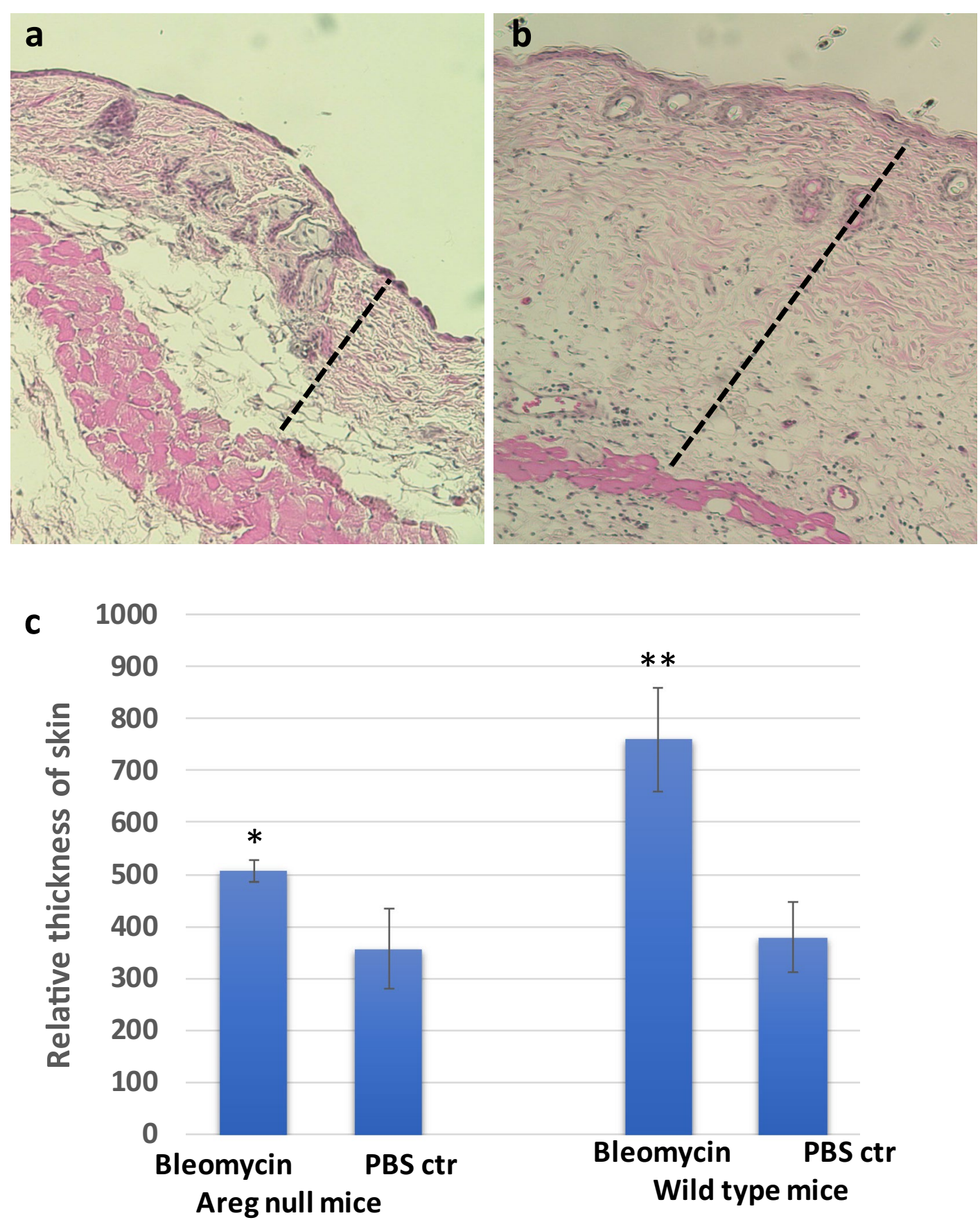

Fig. 2 A significant role of Areg in bleomycin-induced skin fibrosis. $\mathbf{a}$, b show H\&E images of skin biopsies from control mice treated with PBS control (a) or bleomycin (b). c shows a summary of data from 10 mice for each group ( 5 females and 5 males from wild type or Areg knockout). ${ }^{*}$ indicates $<0.05$, and ${ }^{* *}$ indicates $p<0.0001$. Dashed bars indicate skin thickness

result demonstrates that selumetinib is effective in reducing bleomycin-induced skin fibrosis.

We further examined the level of phospho-ERK in selumetinib-treated skin specimens and found that phosphoERK is undetectable after selumetinib treatment (Fig. 4).
Our data using selumetinib further demonstrate that the Arge-EGFR-MEK signaling axis is critical for development of skin fibrosis. 


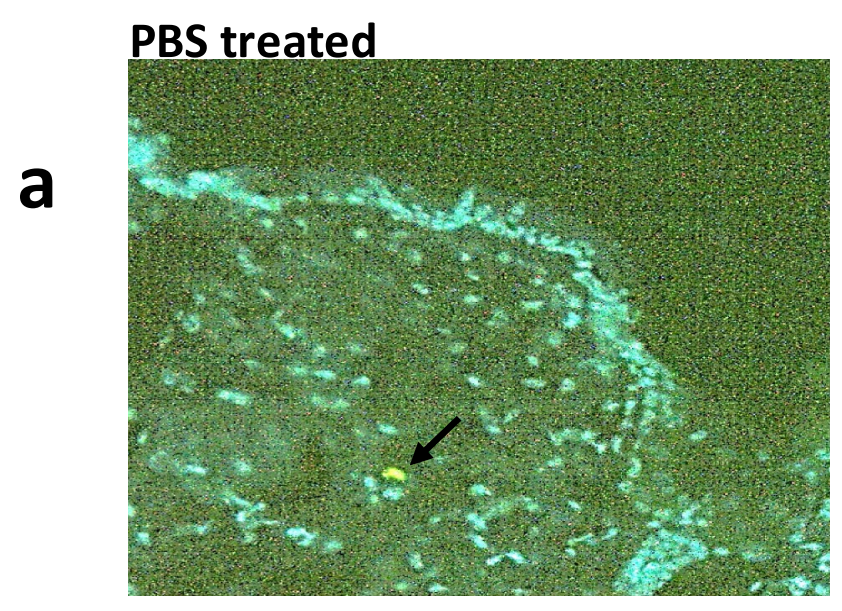

\section{Bleomycin treated}

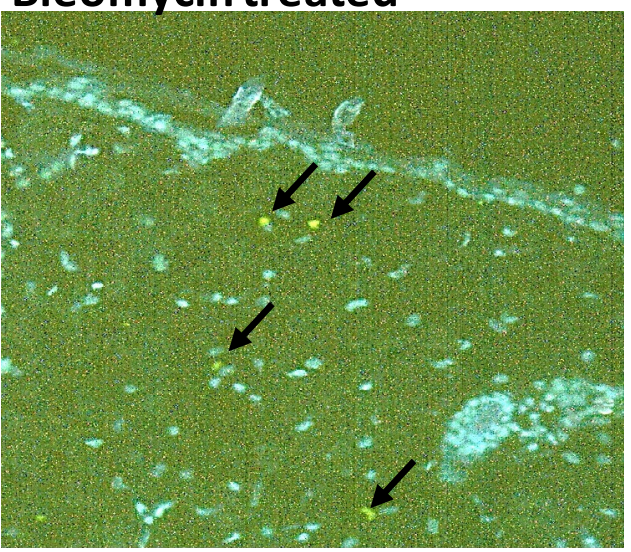

b



Fig. 3 Ki67 positivity in skin biopsies. We detected Ki67 positivity in skin biopsies either treated with PBS or bleomycin through immunofluorescence (IF) staining with ki67 specific antibodies. a shows images with positive Ki67 staining (green, indicated by arrows). b shows a summary of ki67 staining from 6 mice ( 3 females and 3 males) in each group. *indicates $p<0.0001$

\section{Discussion}

Fibrosis is associated with changes in many cell types and a variety of signaling events, many of them interconnected. There are several approaches to dissect the signaling networks critical for the development of fibrosis: (1) analyses of human specimens to determine the signaling changes in fibrosis [25-27]; (2) use of mouse models of fibrosis to dissect changes at different times during fibrosis [28]; (3) comparing different wound healing processes to identify the abnormalities in the fibrosis process [9]. Our approach is to compare the differences between oral mucosa wound healing and skin fibrosis with the assumption that fibrosis is a pathological wound healing process. Thus, identifying the abnormalities in the fibrotic process may help establish new ways to restore the tissue remodeling process. Indeed, we have proved that Areg, a molecule transiently induced in oral wound healing but constitutively upregulated in skin fibrosis, is critical for development of bleomycin-induced skin fibrosis, a hallmark of scleroderma. We further showed that inhibiting downstream signaling of Areg by MEK inhibitor selumetinib is as effective as Areg knockout in prevention of skin fibrosis development. Based on these data, we predict that inhibitors of the Areg-EGFR-MEK signaling axis may be effective in treatment of scleroderma and other fibrotic diseases.

We showed the significance of Areg for skin fibrosis, but the exact mechanisms of action remain to be determined. Using single cell RNA-seq, we generated data to indicate that Areg expresses in at least two cell populations: immune cells and fibroblasts (or myofibroblasts) (Additional file: 4). Further analyses of Areg signaling in these two cell populations will help lineate the signaling network of Areg during skin fibrosis. Previous studies showed that Areg is highly expressed in type 2 innate lymphoid cells (ILC2), which is induced by IL33 [29]. It is also reported that Areg can be regulated by TGFbeta signaling or Hippo-YAP signaling [30-32]. Our single cell 




Fig. 4 ERK-p expression in skin fibrosis and after AZD6244 (selumetinib) treatment. a shows images of nuclear staining with DAPI or ERK-p specific antibodies. The method is described in details in experimental methods. $\mathbf{b}$ is a summary of ERK-p staining using the number of positive cells per field under microscope $(200 \mathrm{X})$. **indicates $\mathrm{p}<0.0001$

RNA-seq showed correlative expression of IL33 and Areg in immune cells, suggesting a role of IL33 in regulation of Areg. Additional analyses of downstream effectors may also help understand Areg signaling during skin fibrosis. Our data with MEK inhibitor selumetinib not only showed the significance of the Areg-EGFR-MEK signaling axis for fibrosis, but also provided novel ways to treat/ prevent fibrosis. Whether blocking the Areg-EGFR-MEK signaling axis is effective in treatment of skin fibrosis will require additional experiment using mice with established skin fibrosis.

While this report is the first to demonstrate the significance of Areg for skin fibrosis, Areg is reported to be associated with hyperproliferative skin diseases in the literature, such as psoriasis [33-39]. In addition, ARGE is also up-regulated in rheumatoid arthritis patients [40]. Indeed, the role of Areg in psoriasis is demonstrated by the facts that AREG transgenic mice show psoriasis-like phenotypes [39]. The significance of Areg in other proliferative skin diseases or other human disorders remains unclear, and the Areg knockout mice may be used for future studies of these diseases.

Are our data relevant to human scleroderma? Gene expression of bulk scleroderma specimens of skin often does not reveal up-regulation of $A R E G$ in the diseased biopsies although a recent report showed up-regulation in scleroderma patients [41]. However, activated immune cells from scleroderma show a high level of Areg expression [42], indicating that expression of $A R E G$ is associated with specific cell types in the disease process, rather than the overall gene expression. Indeed, single cell analyses indicate that $A R E G$ is highly expressed in myofibroblasts, not other types of fibroblasts in scleroderma patients [25]. Additional work is needed to show the relevance of Areg signaling in other types of scleroderma specimens, such as lung and kidney specimens at the single cell level.

During our studies, we also identified several other molecules with similar features as Areg, and their roles in fibrosis have not completed proven. While Fosl1, Mmp9, Cxcl1, Ccl5 and Stat1 have been linked to fibrosis in the literature, their direct evidence for mechanisms of action during fibrosis remain elusive. On the other hand, there are limited resources for studies of Slfn4, Klra2 and Hdc. One approach will be to use gene knockout mice. Knockout mice of Mmp9, Cxcl1, Ccl5, $H d c$ and Stat 1 are viable and may be obtained commercially from Jackson laboratory. Alternatively, neutralizing antibodies to Cxcl1 and Ccl5 can be used to block the function of the targets $[43,44]$. These studies likely generate additional ways to mitigate scleroderma development and progression. 

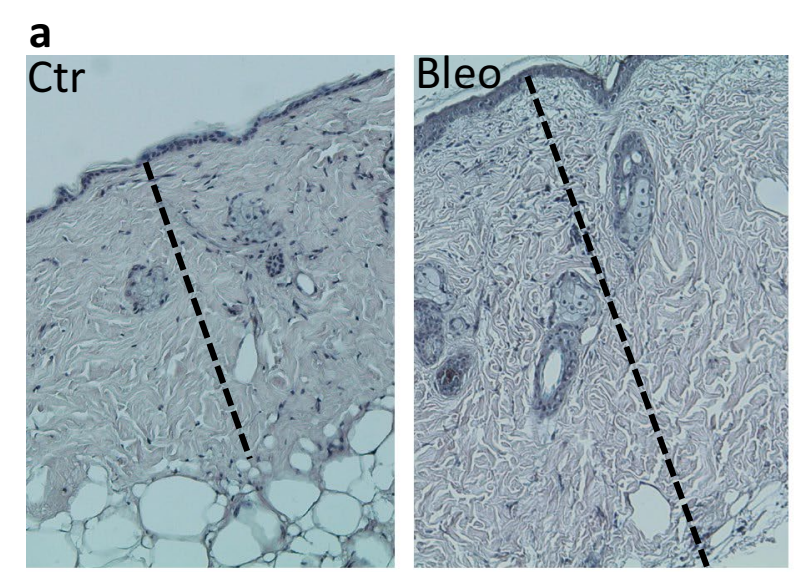

b

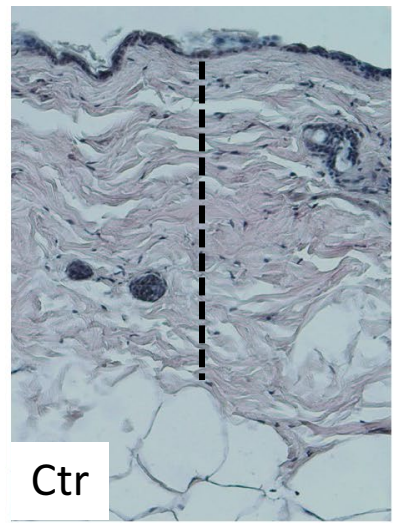

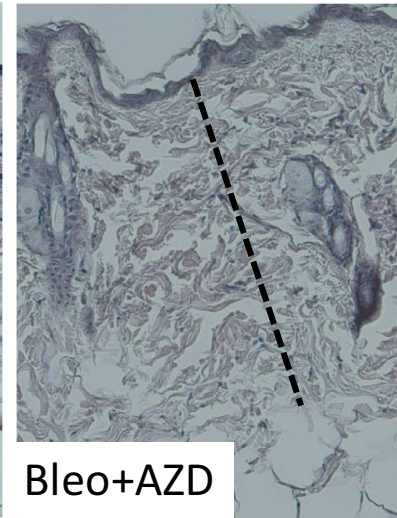

C

\section{Skin thickness}

2.5

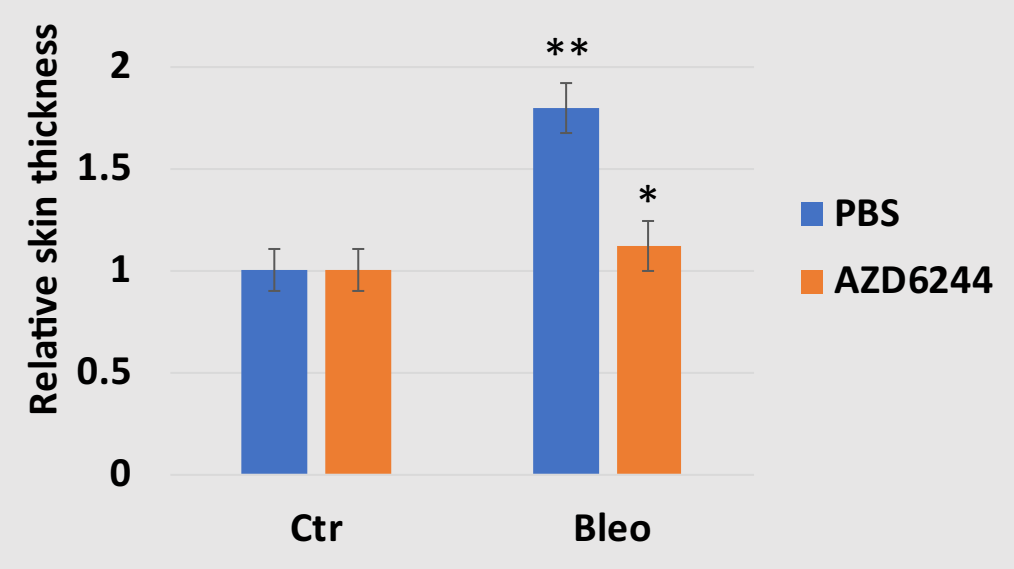

Fig. 5 The effect of MEK inhibitor AZD6244 (shown as AZD, also selumetinib) on bleomycin-induced skin fibrosis. a shows typical H\&E images of skin biopsies from bleomycin-based mouse model of skin fibrosis. $\mathbf{b}$ shows H\&E images from mice treated with AZD6244 during bleomycn-induced skin fibrosis. $\mathbf{c}$ shows the summary of the data from two groups of mice treated with AZD6244 or PBS $(n=3)$. Dashed bars indicate skin thickness. **indicates $p<0.0001$, and *indicates $p=0.045$

\section{Conclusions}

Based on our results, we conclude that Areg and the Areg-EGFR-MEK signaling axis plays an important role in the development of skin fibrosis in bleomycin-based mouse model. Since skin fibrosis is a hallmark of scleroderma, we believe that inhibitors of Areg signaling may be effective in prevention and treatment of scleroderma.

\section{Methods}

\section{Chemicals}

AZD6244 (selumetinib) was purchased as a research agent from the Selleckchem Chemicals LLC (Houston, TX, USA). AZD6244 (selumetinib), originally developed by AstraZeneca, is a highly potent and selective non-ATP competitive inhibitor of MEK with an $\mathrm{IC}_{50}$ of $14 \mathrm{nM}$ [45]. Bleomycin Sulfate, Streptomyces verticillus, was purchased from Sigma (St. Luis, MO).

\section{Animals, bleomycin-based skin fibrosis, and treatment}

Areg knockout and C57Bl/6 mice were purchased from Jackson laboratory (Bar Harbor, ME). Use of animal was approved by the IACUC committee in Indiana University School of Medicine (ethical code 11,370; approval date-15 February 2020). We mated Areg knockout and C57Bl/6 mice over 7 generations to obtain Areg knockout and wild type mice in a similar genetic background. Genotyping of mice was performed by PCR with specific primers provided by the vendors using lysed tail from 
each mouse $[0.3 \mathrm{~cm}$ tail in $100 \mathrm{~mL}$ of PCR Direct (tail) solution (Viagen Inc., Los Angeles, CA) with $1 \mathrm{mg} / \mathrm{mL}$ proteinase $\mathrm{K}$ at $55^{\circ} \mathrm{C}$ overnight, then $85{ }^{\circ} \mathrm{C}$ for $45 \mathrm{~min}$, and use $0.5-1$ microL of the lysate for each 25 microL PCR reaction].

Skin fibrosis was generated according to a previously published protocol [46]. In brief, bleomycin was made in PBS in $1 \mathrm{mg} / \mathrm{ml}$, and intradermal injection was performed using gauge needle \#27 with 50 microL/injection onto the back of mouse skin (at low right side) after fur removal. At up left, we used PBS for injection after fur removal. The procedure was approved by the IUCUC Committee in Indiana University School of Medicine and was strictly followed in the study. We injected mice daily for 10 days before harvesting skin tissues for analyses in skin thickness, gene expression and histology [hematoxylin and eosin (H\&E) staining and Immunofluorescence (IF)]. In bleomycin-induced skin fibrosis studies, we used 10 mice per group (control PBS injection or bleomycin injection, 5 females and 5 males). We used 3 mice for AZD6244 (selumetinib) treatment, and 5 mice for the control group.

AZD6244 was suspended in sterile PBS by sonication at $5 \mathrm{mg} / \mathrm{mL}$. For drug treatment, mice were treated with AZD6244 (oral gavage $10 \mathrm{mg} / \mathrm{kg}$ daily) or vehicle control $(\mathrm{PBS})$ in each group.

\section{Histology, IF staining and tissue measurement}

Histology was performed according to a previously published procedure [47]. Fresh tissue was harvested and fixed with $10 \%$ buffered formalin. Five-micron paraffinembedded sections were labeled with primary antibodies against phosphor-p44/p42 MAPK (Erk1/2) (Thr 202/ Tyr 204) (Cell Signaling Technology Cat\# 4370, 1:200, Danvers, MA, USA), Ki-67 (ab15580, 1:500, AbCam, Cambridge, MA, USA), followed by molecular probe secondary antibodies (Fisher Scientific, Cat\# A11008, 1:300, Grand Island, New York).

Skin histology was viewed after H\&E staining, and the skin thickness was quantified using ImageJ. Skin fibrosis was confirmed by Fast green/Sirius red staining. Fivemicrometer tissue sections were stained with $0.01 \%$ Fast green dissolved in water saturated with picric acid for 15 min $0.1 \%$ Sirius red and 0.04\% Fast green dissolved in water saturated with picric acid for $5 \mathrm{~min}$, dehydrate in $100 \%$ ethanol for $10 \mathrm{~min} \mathrm{[48].}$

To avoid discrepancy from age and genetic backgrounds of the mice, we used littermates from the same mating cage for selection of treatment groups or genotypes. Because of the variation between back skin and abdomen skin in tumor development, we used back skin for injection and histology studies.

\section{RNA extraction, RT-PCR and Real-time PCR}

Total RNAs from tissues were extracted using Tri-RNA reagent from Sigma (St Luis, MO) according to the manufacturer's instruction and 1 microG of total RNA was reverse transcribed into cDNAs using the first-strand synthesis kit (Roche, Tucson, AZ, USA). Real-time quantitative PCR analyses were performed according to a previously published procedure [47]. Triplicate $C T$ values were analyzed in Microsoft Excel using the comparative $\mathrm{Ct}(\mathrm{DDCt})$ method as described by the manufacturer (Applied Biosystems, Foster City, CA, USA). The amount of target (ddCt) was obtained by normalization to an endogenous reference (Gapdh for mice) and relative to a calibrator. All TaqMan primers and probes were purchased from Applied Biosystems Inc.

\section{Single cell RNA-seq, sequence alignment, differential expression analyses}

Tissue was dissociated with Collagenase IV $1 \mathrm{mg} / \mathrm{ml} 37 \mathrm{C}$ for $1 \mathrm{~h}$. Cells were washed with 5\%FBS/PBS twice, each centrifuged $(1000 \mathrm{rpm})$, and dead cells were removed by dead cell removal kit (Miltenyi Biotec, Somerville, MA). Prepared cells were used to perform $10 \times$ Genomics according to a previously published protocol [49]. In brief, appropriate number of cells were loaded on a multiple-channel micro-fluidics chip of the Chromium Single Cell Instrument $(10 \times$ Genomics $)$ with a targeted cell recovery of 9,000. Single cell gel beads in emulsion containing barcoded oligonucleotides and reverse transcriptase reagents were generated with the v3.1 Next GEM Single Cell $3^{\prime}$ reagent kit $(10 \times$ Genomics). Following cell capture and cell lysis, cDNA was synthesized and amplified. Illumina sequencing library was then prepared with the amplified cDNA. The resulting library was sequenced using a custom program on Illumina NovaSeq 6000. $28 \mathrm{bp}$ of cell barcode and UMI sequences and 91 bp RNA reads were generated with Illumina NovaSeq 6000 at CMG of Indiana University School of Medicine. Sequencing analyses were performed as previously reported [50].

\section{Statistical analyses data}

Are presented as mean SD. Statistical analyses were performed using the Mann-Whitney test or the Student $\mathrm{t}$ test (two-tailed) to compare the results, with $\mathrm{P}$ values of $<0.05$ as statistically significant.

\section{Supplementary Information}

The online version contains supplementary material available at https://doi. org/10.1186/s13578-021-00553-0. 
Additional file 1. Ontology analyses of transiently upregulated genes in oral mucosa wound healing.

Additional file 2. The list of genes with transient upregulation in oral mucosa wound healing (The genes in purple are also upregulated in bleomycin-induced skin fibrosis).

Additional file 3. Sirius red/fast green staining of skin tissues.

Additional file 4. A summary of single cell RNAseq analyses.

\section{Abbreviations}

AREG: Amphiregulin; ECM: Extracellular matrix; MEK: MAPK/ERK kinase, ERKextracellular receptor-stimulated kinase, MAPK- mitogen-activated protein kinase; EGFR: Epidermal Growth Factor Receptor; H\&E: Hematoxylin and Eosin; ILC2: Group 2 innate lymphoid cells; IL33: Interleukin 33.

\section{Acknowledgement}

We thank The Well Center for Pediatric Research, Jeff Gordon Research Laboratory, AGA, Healthcare Initiatives, Inc. and CTSI Indiana for support. Single cell analysis was carried out in the Center for Medical Genomics at Indiana University School of Medicine, which is partially supported by the Indiana University Grand Challenges Precision Health Initiative. This work was supported by the Riley Foundation for Children, AGA and Indiana University School of Medicine Biomedical Research Grant.

\section{Authors' contributions}

Conceptualization and Design: JX. Resources: JX. Methodology: MZ, SF, XZ, GD. Data Analysis and Interpretation: HG, JW, YL, JX. Writing, review, and/or revision of the manuscript: JX. Supervision: JX. All authors read and approved the final manuscript.

\section{Funding}

Not applicable.

\section{Availability of data and materials}

Not applicable.

\section{Ethics approval and consent to participate}

Use of animal was approved by the IACUC committee in Indiana University School of Medicine (ethical code 11370; approval date-15 February 2020).

\section{Consent to publication}

Not applicable.

\section{Competing interests}

The authors declare that they have no conflict of interest.

\section{Author details}

${ }^{1}$ Department of Pediatrics, The Wells Center for Pediatric Research, Indiana University School of Medicine, Indianapolis, IN, USA. ${ }^{2}$ Department of BioHealth Informatics, School of Informatics and Computing At IUPUI, Indiana University, Indianapolis, IN, USA. ${ }^{3}$ The IU Simon Comprehensive Cancer Center, Indiana University, Indianapolis, IN, USA. ${ }^{4}$ The Center for Computational Biology and Bioinformatics, Indiana University School of Medicine, Indianapolis, IN, USA. ${ }^{5}$ Department of Medical and Molecular Genetics, Indiana University School of Medicine, Indianapolis, IN, USA.

Received: 18 January 2021 Accepted: 10 February 2021

Published online: 23 February 2021

\section{References}

1. Ingegnoli F, Ughi N, Mihai C. Update on the epidemiology, risk factors, and disease outcomes of systemic sclerosis. Best Pract Res Clin Rheumatol. 2018;32:223-40.

2. Barnes J, Mayes MD. Epidemiology of systemic sclerosis: incidence, prevalence, survival, risk factors, malignancy, and environmental triggers. Curr Opin Rheumatol. 2012;24:165-70.
3. Distler JHW, Gyorfi AH, Ramanujam M, Whitfield ML, Konigshoff M, Lafyatis R. Shared and distinct mechanisms of fibrosis. Nat Rev Rheumatol. 2019:15:705-30.

4. Nanthakumar CB, Hatley RJ, Lemma S, Gauldie J, Marshall RP, Macdonald SJ. Dissecting fibrosis: therapeutic insights from the small-molecule toolbox. Nat Rev Drug Discov. 2015;14:693-720.

5. Rockey DC, Bell PD, Hill JA. Fibrosis-A Common Pathway to Organ Injury and Failure. N Engl J Med. 2015;373:96.

6. Wynn TA. Cellular and molecular mechanisms of fibrosis. J Pathol. 2008;214:199-210.

7. Wynn TA. Fibrotic disease and the $T(H) 1 / T(H) 2$ paradigm. Nat Rev Immunol. 2004;4:583-94.

8. Glim JE, van Egmond M, Niessen FB, Everts V, Beelen RH. Detrimental dermal wound healing: what can we learn from the oral mucosa? Wound Repair Regen. 2013;21:648-60.

9. Chen L, Arbieva ZH, Guo S, Marucha PT, Mustoe TA, DiPietro LA. Positional differences in the wound transcriptome of skin and oral mucosa. BMC Genomics. 2010;11:471.

10. Leonardo TR, Shi J, Chen D, Trivedi HM, Chen L. Differential Expression and Function of Bicellular Tight Junctions in Skin and Oral Wound Healing. Int J Mol Sci 2020;21.

11. Vafashoar F, Mousavizadeh K, Poormoghim H, et al. Gelatinases Increase in Bleomycin-induced Systemic Sclerosis Mouse Model. Iran J Allergy Asthma Immunol. 2019;18:182-9.

12. Kim WU, Min SY, Cho ML, et al. Elevated matrix metalloproteinase-9 in patients with systemic sclerosis. Arthritis Res Ther. 2005;7:R71-9.

13. Waszczykowska A, Podgorski M, Waszczykowski M, Gerlicz-Kowalczuk Z, Jurowski P. Matrix Metalloproteinases MMP-2 and MMP-9, Their Inhibitors TIMP-1 and TIMP-2, Vascular Endothelial Growth Factor and sVEGFR-2 as Predictive Markers of Ischemic Retinopathy in Patients with Systemic Sclerosis-Case Series Report. Int J Mol Sci 2020;21.

14. Matsuda KM, Yoshizaki A, Kuzumi A, et al. Skin thickness score as a surrogate marker of organ involvements in systemic sclerosis: a retrospective observational study. Arthritis Res Ther. 2019;21:129.

15. Amjadi S, Maranian P, Furst DE, et al. Course of the modified Rodnan skin thickness score in systemic sclerosis clinical trials: analysis of three large multicenter, double-blind, randomized controlled trials. Arthritis Rheum. 2009:60:2490-8.

16. Clements PJ, Lachenbruch PA, Ng SC, Simmons M, Sterz M, Furst DE. Skin score. A semiquantitative measure of cutaneous involvement that improves prediction of prognosis in systemic sclerosis. Arthritis Rheum 1990;33:1256-63.

17. Zheng B, Nevskaya T, Baxter CA, et al. Changes in skin score in early diffuse cutaneous systemic sclerosis are associated with changes in global disease severity. Rheumatology (Oxford). 2020;59:398-406.

18. He Y, Tsou PS, Khanna D, Sawalha AH. Methyl-CpG-binding protein 2 mediates antifibrotic effects in scleroderma fibroblasts. Ann Rheum Dis. 2018;77:1208-18.

19. Mikamo M, Kitagawa K, Sakai S, et al. Inhibiting Skp2 E3 Ligase Suppresses Bleomycin-Induced Pulmonary Fibrosis. Int J Mol Sci 2018;19.

20. Fan Y, Zhang W, Wei H, Sun R, Tian Z, Chen Y. Hepatic NK cells attenuate fibrosis progression of non-alcoholic steatohepatitis in dependent of CXCL10-mediated recruitment. Liver Int. 2020;40:598-608.

21. Kendall RT, Feghali-Bostwick CA. Fibroblasts in fibrosis: novel roles and mediators. Front Pharmacol. 2014;5:123.

22. Bergqvist C, Wolkenstein P. MEK inhibitors in RASopathies. Curr Opin Oncol 2020;Publish Ahead of Print.

23. Killock D. Selumetinib benefits children with inoperable plexiform neurofibromas. Nat Rev Clin Oncol. 2020;17:273.

24. Gross AM, Wolters PL, Dombi E, et al. Selumetinib in Children with Inoperable Plexiform Neurofibromas. N Engl J Med. 2020;382:1430-42.

25. Valenzi E, Bulik M, Tabib T, et al. Single-cell analysis reveals fibroblast heterogeneity and myofibroblasts in systemic sclerosis-associated interstitial lung disease. Ann Rheum Dis. 2019;78:1379-87.

26. Lu T, Klein KO, Colmegna I, Lora M, Greenwood CMT, Hudson M. Wholegenome bisulfite sequencing in systemic sclerosis provides novel targets to understand disease pathogenesis. BMC Med Genomics. 2019;12:144.

27. Apostolidis SA, Stifano G, Tabib T, et al. Single Cell RNA Sequencing Identifies HSPG2 and APLNR as Markers of Endothelial Cell Injury in Systemic Sclerosis Skin. Front Immunol. 2018;9:2191. 
28. Yue X, Yu X, Petersen F, Riemekasten G. Recent advances in mouse models for systemic sclerosis. Autoimmun Rev. 2018;17:1225-34.

29. Cao Q, Wang Y, Niu Z, et al. Potentiating Tissue-Resident Type 2 Innate Lymphoid Cells by IL-33 to Prevent Renal Ischemia-Reperfusion Injury. J Am Soc Nephrol. 2018;29:961-76.

30. Chen F, Yang W, Huang $X$, et al. Neutrophils Promote Amphiregulin Production in Intestinal Epithelial Cells through TGF-beta and Contribute to Intestinal Homeostasis. J Immunol. 2018;201:2492-501.

31. Zhang J, Ji JY, Yu M, et al. YAP-dependent induction of amphiregulin identifies a non-cell-autonomous component of the Hippo pathway. Nat Cell Biol. 2009;11:1444-50.

32. Lefort S, Tan S, Balani S, et al. Initiation of human mammary cell tumorigenesis by mutant KRAS requires YAP inactivation. Oncogene. 2020;39:1957-68.

33. Piepkorn M, Pittelkow MR, Cook PW. Autocrine regulation of keratinocytes: the emerging role of heparin-binding, epidermal growth factorrelated growth factors. J Invest Dermatol. 1998;111:715-21.

34. Piepkorn M. Overexpression of amphiregulin, a major autocrine growth factor for cultured human keratinocytes, in hyperproliferative skin diseases. Am J Dermatopathol. 1996;18:165-71.

35. Yoshida A, Kanno H, Watabe D, Akasaka T, Sawai T. The role of heparinbinding EGF-like growth factor and amphiregulin in the epidermal proliferation of psoriasis in cooperation with TNFalpha. Arch Dermatol Res. 2008;300:37-45.

36. Chung E, Cook PW, Parkos CA, Park YK, Pittelkow MR, Coffey RJ. Amphiregulin causes functional downregulation of adherens junctions in psoriasis. J Invest Dermatol. 2005;124:1134-40.

37. Cook PW, Brown JR, Cornell KA, Pittelkow MR. Suprabasal expression of human amphiregulin in the epidermis of transgenic mice induces a severe, early-onset, psoriasis-like skin pathology: expression of amphiregulin in the basal epidermis is also associated with synovitis. Exp Dermatol. 2004;13:347-56.

38. Cook PW, Pittelkow MR, Piepkorn M. Overexpression of amphiregulin in the epidermis of transgenic mice induces a psoriasis-like cutaneous phenotype. J Invest Dermatol. 1999;113:860.

39. Cook PW, Piepkorn M, Clegg CH, et al. Transgenic expression of the human amphiregulin gene induces a psoriasis-like phenotype. J Clin Invest. 1997;100:2286-94.
40. Yamane S, Ishida S, Hanamoto Y, et al. Proinflammatory role of amphiregulin, an epidermal growth factor family member whose expression is augmented in rheumatoid arthritis patients. J Inflamm (Lond). 2008;5:5.

41. Kobayashi S, Nagafuchi Y, Okubo M, et al. Integrated bulk and singlecell RNA-sequencing identified disease-relevant monocytes and a gene network module underlying systemic sclerosis. J Autoimmun. 2021;116:102547.

42. Gao X, Jia G, Guttman A, et al. Osteopontin Links Myeloid Activation and Disease Progression in Systemic Sclerosis. Cell Rep Med. 2020;1:100140.

43. Casasanta MA, Yoo CC, Udayasuryan B, et al. Fusobacterium nucleatum host-cell binding and invasion induces IL-8 and CXCL1 secretion that drives colorectal cancer cell migration. Sci Signal 2020;13.

44. Amorim NRT, Souza-Almeida G, Luna-Gomes T, et al. Leptin Elicits In Vivo Eosinophil Migration and Activation: Key Role of Mast Cell-Derived PGD2. Front Endocrinol (Lausanne). 2020;11:572113.

45. Yeh TC, Marsh V, Bernat BA, et al. Biological characterization of ARRY142886 (AZD6244), a potent, highly selective mitogen-activated protein kinase kinase 1/2 inhibitor. Clin Cancer Res. 2007;13:1576-83.

46. Blyszczuk P, Kozlova A, Guo Z, Kania G, Distler O. Experimental Mouse Model of Bleomycin-Induced Skin Fibrosis. Curr Protoc Immunol. 2019;126:e88

47. Fan Q, Gu D, Liu H, et al. Defective TGF-beta signaling in bone marrowderived cells prevents hedgehog-induced skin tumors. Cancer Res. 2014;74:471-83.

48. Sato M, Mikamo A, Kurazumi H, et al. Ratio of preoperative atrial natriuretic peptide to brain natriuretic peptide predicts the outcome of the maze procedure in mitral valve disease. J Cardiothorac Surg. 2013;8:32.

49. Shao X, Liao J, Lu X, Xue R, Ai N, Fan X. scCATCH: Automatic Annotation on Cell Types of Clusters from Single-Cell RNA Sequencing Data. iscience 2020;23:100882.

50. Gu D, Lin H, Zhang $X$, et al. Simultaneous Inhibition of MEK and Hh Signaling Reduces Pancreatic Cancer Metastasis. Cancers (Basel) 2018;10.

\section{Publisher's Note}

Springer Nature remains neutral with regard to jurisdictional claims in published maps and institutional affiliations.
Ready to submit your research? Choose BMC and benefit from:

- fast, convenient online submission

- thorough peer review by experienced researchers in your field

- rapid publication on acceptance

- support for research data, including large and complex data types

- gold Open Access which fosters wider collaboration and increased citations

- maximum visibility for your research: over 100M website views per year

At BMC, research is always in progress.

Learn more biomedcentral.com/submissions 